\title{
Infinitely many nonradial solutions for the Hénon equation with critical growth
}

\author{
Juncheng Wei and Shusen Yan
}

Abstract. We consider the following Hénon equation with critical growth:

$$
(*) \begin{cases}-\Delta u=|y|^{\alpha} u^{\frac{N+2}{N-2}}, u>0 & y \in B_{1}(0), \\ u=0, & \text { on } \partial B_{1}(0),\end{cases}
$$

where $\alpha>0$ is a positive constant, $B_{1}(0)$ is the unit ball in $\mathbb{R}^{N}$, and $N \geq 4$. Ni [9] proved the existence of a radial solution and Serra [12] proved the existence of a nonradial solution for $\alpha$ large and $N \geq 4$. In this paper, we show the existence of a nonradial solution for any $\alpha>0$ and $N \geq 4$. Furthermore, we prove that equation $(*)$ has infinitely many nonradial solutions, whose energy can be made arbitrarily large.

\section{Introduction}

Of concern is the following Hénon equation with critical growth:

$$
\begin{cases}-\Delta u=|y|^{\alpha} u^{\frac{N+2}{N-2}}, u>0, & y \in B_{1}(0), \\ u=0, & \text { on } \partial B_{1}(0),\end{cases}
$$

where $\alpha>0$ is a positive constant, $B_{1}(0)$ is the unit ball in $\mathbb{R}^{N}$, and $N \geq 3$.

Equation (1.1) arises in the study of astrophysics, see [7]. If the exponent $(N+2) /(N-2)$ is replaced by $p$, where $p<(N+2) /(N-2)$, a solution can be obtained easily by variational methods. When $p=(N+2) /(N-2)$, the loss of compactness from $H_{0}^{1}\left(B_{1}(0)\right)$ to $L^{\frac{2 N}{N-2}}\left(B_{1}(0)\right)$ makes the problem (1.1) very difficult to study. $\mathrm{Ni}[9]$ first proved the existence of a radial solution for any $\alpha>0$. On the other hand, it is easy to check that the mountain pass value $c$ corresponding to $(1.1)$ is

$$
c=\frac{1}{N} S^{N / 2}
$$

Mathematics Subject Classification (2010): Primary 35J65; Secondary 35B38, 47J30.

Keywords: Henon's equation, infinitely many solutions, critical Sobolev exponent, reduction method. 
where $S$ is best Sobolev constant of the embedding from $D^{1,2}\left(\mathbb{R}^{N}\right)$ to $L^{\frac{2 N}{N-2}}\left(\mathbb{R}^{N}\right)$, from which we can deduce that $c$ is not a critical value of the functional corresponding to (1.1). When $N=2$, Smets-Su-Willem [13] showed that the mountain pass solution is nonradial when $\alpha$ is large. When $N \geq 3$, for the Hénon equations with nearly critical growth (replacing $(N+2) /(N-2)$ in $(1.1)$ by $(N+2) /(N-2)-\varepsilon$ with $\varepsilon>0$ small), Cao-Peng [3] proved that the mountain pass solution is nonradial and blows up as $\varepsilon \rightarrow 0$. Thus, it is natural to ask whether (1.1) has a nonradial solution. Using a variational method, Serra [12] proved that (1.1) has a nonradial solution when $N \geq 4$ and $\alpha$ is large. As far as we know, up to now, there is no result showing the existence of nonradial solution of (1.1), nor is there a multiplicity result, with arbitrary $\alpha>0$, for (1.1).

The aim of this paper is to prove that (1.1) has infinitely many nonradial solutions if $N \geq 4$. In fact, we will study a more general problem:

$$
\begin{cases}-\Delta u=K(|y|) u^{\frac{N+2}{N-2}}, u>0, & y \in B_{1}(0), \\ u=0, & \text { on } \partial B_{1}(0),\end{cases}
$$

where $K(r)$ is a bounded function defined in [0,1]. It is easy to see that a necessary condition for the existence of a solution of (1.2) is that $K(r)$ is positive somewhere. On the other hand, Pohozaev identity implies (1.2) has no solution if $K^{\prime}(r) \leq 0$ in $[0,1]$. Concerning the existence of solutions for (1.2), using the same method as in $[15]$, we can prove the following existence result:

Theorem A. Suppose that there is a $r_{0} \in(0,1)$, such that $K\left(r_{0}\right)>0$, and

$$
K(r)=K\left(r_{0}\right)-K_{0}\left|r-r_{0}\right|^{m}+O\left(\left|r-r_{0}\right|^{m+\theta}\right), \quad \text { as } r \rightarrow r_{0},
$$

where $m \in[2, N-2), K_{0}>0$, and $\theta>0$ are some constants. Then, for $N \geq 5$, the problem (1.2) has infinitely many nonradial solutions.

Note that for the Hénon equation, $K(r)=r^{\alpha}$, which has no critical point in $(0,1)$. So, Theorem A does not apply to the Henon equation (1.1).

Condition (1.3) implies that $r_{0}$ is a local maximum point of $K(r)$, and thus a critical point of $K(r)$. The function $r^{\alpha}$ attains its maximum on $[0,1]$ at $r_{0}=1$, but $r_{0}=1$ is not a critical point of $r^{\alpha}$.

The aim of this paper is to show that if $K(r)$ is increasing near $r_{0}=1$ (so it is a maximum point of $K(r)$ on $[1-\delta, 1]$ for some small $\delta>0)$, the zero Dirichlet boundary condition makes it possible to construct infinitely many solutions of (1.2), although $r_{0}=1$ is not a critical point of $K(r)$. Our main result in this paper can be stated as follows:

Theorem 1.1. Suppose that $N \geq 4$. If $K(r)$ satisfies $K(1)>0$ and $K^{\prime}(1)>0$, then problem (1.2))has infinitely many nonradial solutions. In particular, the Hénon equation (1.1) has infinitely many nonradial solutions.

Recall that a necessary condition for the existence of at least one solution of $(1.2)$ is that $K^{\prime}(r)$ is positive somewhere on [0,1]. If $K(r) \geq 0$ and $N \geq 5$, Theorems A and 1.1 show that under a condition which is slightly stronger than this necessary condition, (1.2) has infinitely many solutions. 
We think that the condition that $N \geq 4$ is just technical. The reason is that the reduced energy does have a critical point when $N=3$. The problem lies in the reduction part which should be only technical. (Some partial (negative) results are obtained by O. Druet and Laurain [6].).

The reader can refer to [1], [2], [4], [8], [10], [11], and [14] for results on Hénon equations involving subcritical and near critical exponents.

Before we close this introduction, let us outline the main idea in the proof of Theorem 1.1.

Let us fix a positive integer $k \geq k_{0}$, where $k_{0}$ is large, which is to be determined later.

Set

$$
\mu=k^{\frac{N-1}{N-2}}, \quad N \geq 4
$$

to be the scaling parameter.

Let $2^{*}=2 N /(N-2)$. Using the transformation $u(y) \mapsto \mu^{-(N-2) / 2} u(y / \mu)$, we find that (1.2) becomes

$$
\begin{cases}-\Delta u=K\left(\frac{|y|}{\mu}\right) u^{2^{*}-1}, u>0, & y \in B_{\mu}(0), \\ u=0, & \text { on } \partial B_{\mu}(0) .\end{cases}
$$

It is well known that the functions

$$
U_{x, \Lambda}(y)=(N(N-2))^{(N-2) / 4}\left(\frac{\Lambda}{1+\Lambda^{2}|y-x|^{2}}\right)^{(N-2) / 2}, \quad \mu>0, \quad x \in \mathbb{R}^{N}
$$

are the only solutions to the following problem

$$
-\Delta u=u^{\frac{N+2}{N-2}}, \quad u>0 \text { in } \mathbb{R}^{N} .
$$

As the scaling parameter $\Lambda \rightarrow+\infty, U_{x, \Lambda}$ is called a single-bubble centered at the point $x$. Since there is no small parameter in (1.1) (here $\mu$ is fixed), we use the scaling parameter $\Lambda$ as the blow-up parameter. Our main idea is to place a large number of bubbles inside $\Omega$. Then the scaling parameter will be determined by the number of bubbles. We put many bubbles along a $k$-polygon inside the domain $B_{1}(0)$ but near the boundary. See Figure 1. (The idea of using the number of bubbles as parameter was first introduced in [15].)

Let us remark that the variational method of Serra [12] also uses the dihedral symmetry of $k$-polygons. By using the $D_{k} \times O(N-2)$ symmetry, the problem $(1.1)$ can be reduced to the one in a sector. He then showed that under dihedral symmetry, the loss of compactness can be recovered if the critical value is below some constant, which holds true when $N \geq 4$. To show that the solution is nonradial, he needed to compare with the energy level of a radial solution. There the condition that $\alpha$ is large is needed. Our method of construction is direct and gives more information.

We continue our construction. Since $U_{x, \Lambda}$ is not zero on $\partial B_{\mu}(0)$, we define $P U_{x, \Lambda}$ as the solution of the following problem:

$$
\Delta P U_{x, \Lambda}=\Delta U_{x, \Lambda}, \quad \text { in } B_{\mu}(0), \quad \Delta P U_{x, \Lambda}=0 \quad \text { on } \partial B_{\mu}(0) .
$$




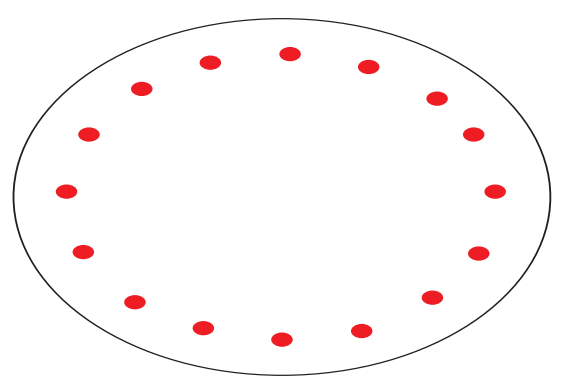

Figure 1. The location of the bubbles.

Let $y=\left(y^{\prime}, y^{\prime \prime}\right), y^{\prime} \in \mathbb{R}^{2}, y^{\prime \prime} \in \mathbb{R}^{N-2}$. Define

$$
\begin{aligned}
H_{s}=\{u: & u \in H_{0}^{1}\left(B_{\mu}(0)\right), u \text { is even in } y_{h}, h=2, \ldots, N, \\
& \left.u\left(r \cos \theta, r \sin \theta, y^{\prime \prime}\right)=u\left(r \cos \left(\theta+\frac{2 \pi j}{k}\right), r \sin \left(\theta+\frac{2 \pi j}{k}\right), y^{\prime \prime}\right)\right\} .
\end{aligned}
$$

Let

$$
x_{j}=\left(r \cos \frac{2(j-1) \pi}{k}, r \sin \frac{2(j-1) \pi}{k}, 0\right), \quad j=1, \ldots, k,
$$

where 0 is the zero vector in $\mathbb{R}^{N-2}$, and let

$$
W_{r, \Lambda}(y)=\sum_{j=1}^{k} P U_{x_{j}, \Lambda} .
$$

In this paper, we always assume that

$$
r \in\left[\mu\left(1-\frac{r_{0}}{k}\right), \mu\left(1-\frac{r_{1}}{k}\right)\right], \quad \text { for some constants } r_{1}>r_{0}>0,
$$

and

$$
L_{0} \leq \Lambda \leq L_{1}, \quad \text { for some constants } L_{1}>L_{0}>0 .
$$

Theorem 1.1 is a direct consequence of the following result:

Theorem 1.2. Suppose that $N \geq 4$. If $K(1)>0$ and $K^{\prime}(1)>0$, then there is an integer $k_{0}>0$, such that for any integer $k \geq k_{0}$, (1.4) has a solution $u_{k}$ of the form

$$
u_{k}=W_{r_{k}, \Lambda_{k}}(y)+\omega_{k},
$$

where $\omega_{k} \in H_{s}$, and as $k \rightarrow+\infty,\left\|\omega_{k}\right\|_{L^{\infty}} \rightarrow 0, L_{0} \leq \Lambda_{k} \leq L_{1}$, and $r_{k} \in$ $\left(\mu\left(1-r_{0} / k\right), \mu\left(1-r_{1} / k\right)\right)$.

Unlike Theorem A, where the result was proved by constructing solutions with many bubbles near the local maximum point $r_{0} \in(0,1)$, the solutions constructing in Theorem 1.1 have many bubbles near the boundary of the unit ball $B_{1}(0)$. In Theorem 1.1, $r_{0}=1$ is not a critical point of $K(r)$ anymore. It is the zero boundary condition that plays a very important role in the construction of solutions with many bubbles near $|y|=1$. 


\section{Finite-dimensional reduction}

In this section, we perform a finite-dimensional reduction. Let

$$
\|u\|_{*}=\sup _{y \in B_{\mu}(0)}\left(\sum_{j=1}^{k} \frac{1}{\left(1+\left|y-x_{j}\right|\right)^{\frac{N-2}{2}+\tau}}\right)^{-1}|u(y)|,
$$

and

$$
\|f\|_{* *}=\sup _{y \in B_{\mu}(0)}\left(\sum_{j=1}^{k} \frac{1}{\left(1+\left|y-x_{j}\right|\right)^{\frac{N+2}{2}+\tau}}\right)^{-1}|f(y)|,
$$

where $\tau=(N-2) /(N-1)$ if $N \geq 4$. For this choice of $\tau$, we find that

$$
\sum_{j=2}^{k} \frac{1}{\left|x_{j}-x_{1}\right|^{\tau}} \leq \frac{C k^{\tau}}{\mu^{\tau}} \sum_{j=2}^{k} \frac{1}{j^{\tau}} \leq \frac{C k}{\mu^{\tau}} \leq C^{\prime} .
$$

Let

$$
Z_{i, 1}=\frac{\partial P U_{x_{i}, \Lambda}}{\partial r}, \quad Z_{i, 2}=\frac{\partial P U_{x_{i}, \Lambda}}{\partial \Lambda}
$$

Consider

$$
\left\{\begin{array}{l}
-\Delta \phi_{k}-\left(2^{*}-1\right) K\left(\frac{|y|}{\mu}\right) W_{r, \Lambda}^{2^{*}-2} \phi_{k}=h+\sum_{j=1}^{2} c_{j} \sum_{i=1}^{k} U_{x_{i}, \Lambda}^{2^{*}-2} Z_{i, j}, \quad \text { in } B_{\mu}(0) \\
\phi_{k} \in H_{s} \\
<U_{x_{i}, \Lambda}^{2^{*}-2} Z_{i, l}, \phi_{k}>=0, \quad i=1, \ldots, k, l=1,2
\end{array}\right.
$$

for some numbers $c_{i}$, where $\langle u, v\rangle=\int_{B_{\mu}(0)} u v$.

Lemma 2.1. Assume that $\phi_{k}$ solves (2.3) for $h=h_{k}$. If $\left\|h_{k}\right\|_{* *}$ goes to zero as $k$ goes to infinity, so does $\left\|\phi_{k}\right\|_{*}$.

Proof. The proof of this lemma is similar to the proof of Lemma 2.1 in [15]. Therefore, we only sketch it.

We argue by contradiction. Suppose that there are $k \rightarrow+\infty, h=h_{k}, \Lambda_{k} \in$ $\left[L_{1}, L_{2}\right], r_{k} \in\left[\mu\left(1-r_{0} / k\right), \mu\left(1-r_{1} / k\right)\right]$, and $\phi_{k}$ solving $(2.3)$ for $h=h_{k}, \Lambda=\Lambda_{k}$, and $r=r_{k}$, with $\left\|h_{k}\right\|_{* *} \rightarrow 0$, and $\left\|\phi_{k}\right\|_{*} \geq c^{\prime}>0$. We may assume that $\left\|\phi_{k}\right\|_{*}=1$. For simplicity, we drop the subscript $k$.

We rewrite (2.3) as

$$
\begin{aligned}
\phi(y)= & \left(2^{*}-1\right) \int_{B_{\mu}(0)} \frac{1}{|z-y|^{N-2}} K\left(\frac{|z|}{\mu}\right) W_{r, \Lambda}^{2^{*}-2} \phi(z) d z \\
& +\int_{B_{\mu}(0)} \frac{1}{|z-y|^{N-2}}\left(h(z)+\sum_{j=1}^{2} c_{j} \sum_{i=1}^{k} Z_{i, j}(z) U_{x_{i}, \Lambda}^{2^{*}-2}(z)\right) d z .
\end{aligned}
$$


Using Lemma B.3, we have

$$
\begin{aligned}
& \left|\left(2^{*}-1\right) \int_{B_{\mu}(0)} \frac{1}{|z-y|^{N-2}} K\left(\frac{|z|}{\mu}\right) W_{r, \Lambda}^{2^{*}-2} \phi(z) d z\right| \\
& \quad \leq C\|\phi\|_{*} \int_{B_{\mu}(0)} \frac{1}{|z-y|^{N-2}} W_{r, \Lambda}^{2^{*}-2} \sum_{j=1}^{k} \frac{1}{\left(1+\left|z-x_{j}\right|\right)^{\frac{N-2}{2}+\tau}} d z \\
& \quad \leq C\|\phi\|_{*} \sum_{j=1}^{k} \frac{1}{\left(1+\left|y-x_{j}\right|\right)^{\frac{N-2}{2}+\tau+\theta}} .
\end{aligned}
$$

It follows from Lemma B.2 that

$$
\left|\int_{B_{\mu}(0)} \frac{1}{|z-y|^{N-2}} h(z) d z\right| \leq C\|h\|_{* *} \sum_{j=1}^{k} \frac{1}{\left(1+\left|y-x_{j}\right|\right)^{\frac{N-2}{2}+\tau}} .
$$

and

$$
\left|\int_{B_{\mu}(0)} \frac{1}{|z-y|^{N-2}} \sum_{i=1}^{k} Z_{i, l}(z) U_{x_{i}, \Lambda}^{2^{*}-2}(z) d z\right| \leq C \sum_{i=1}^{k} \frac{1}{\left(1+\left|y-x_{i}\right|\right)^{\frac{N-2}{2}+\tau}} .
$$

Next, we estimate $c_{l}, l=1,2$. Multiplying (2.3) by $Z_{1, l}$ and integrating, we see that $c_{t}$ satisfies

$$
\sum_{t=1}^{2} \sum_{i=1}^{k}\left\langle U_{x_{i}, \Lambda}^{2^{*}-2} Z_{i, t}, Z_{1, l}\right\rangle c_{t}=\left\langle-\Delta \phi-\left(2^{*}-1\right) K\left(\frac{|y|}{\mu}\right) W_{r, \Lambda}^{2^{*}-2} \phi, Z_{1, l}\right\rangle-\left\langle h, Z_{1, l}\right\rangle
$$

It follows from Lemma B.1 that

$$
\left|\left\langle h, Z_{1, l}\right\rangle\right| \leq C\|h\|_{* *} \int_{\mathbb{R}^{N}} \frac{1}{\left(1+\left|z-x_{1}\right|\right)^{N-2}} \sum_{j=1}^{k} \frac{1}{\left(1+\left|z-x_{j}\right|\right)^{\frac{N+2}{2}+\tau}} d z \leq C\|h\|_{* *} .
$$

On the other hand, using Lemma B.3, we can prove

$$
\begin{aligned}
\langle-\Delta \phi- & \left.\left(2^{*}-1\right) K\left(\frac{|z|}{\mu}\right) W_{r, \Lambda}^{2^{*}-2} \phi, Z_{1, l}\right\rangle \\
= & \left(2^{*}-1\right)\left\langle\left(1-K\left(\frac{|z|}{\mu}\right) W_{r, \Lambda}^{2^{*}-2} Z_{1, l}, \phi\right\rangle=o\left(\|\phi\|_{*}\right) .\right.
\end{aligned}
$$

However, there is a constant $\bar{c}>0$,

$$
\sum_{i=1}^{k}\left\langle U_{x_{i}, \Lambda}^{2^{*}-2} Z_{i, t}, Z_{1, l}\right\rangle=(\bar{c}+o(1)) \delta_{t l}
$$

Thus we obtain from (2.8) that

$$
c_{l}=o\left(\|\phi\|_{*}\right)+O\left(\|h\|_{* *}\right) .
$$


So,

$$
\|\phi\|_{*} \leq\left(o(1)+\left\|h_{k}\right\|_{* *}+\frac{\sum_{j=1}^{k}\left(1+\left|y-x_{j}\right|\right)^{-\frac{N-2}{2}-\tau-\theta}}{\sum_{j=1}^{k}\left(1+\left|y-x_{j}\right|\right)^{-\frac{N-2}{2}-\tau}}\right) .
$$

Since $\|\phi\|_{*}=1$, we obtain from (2.11) that there is $R>0$, such that

$$
\|\phi(y)\|_{B_{R}\left(x_{i}\right)} \geq a>0,
$$

for some $i$. However, $\bar{\phi}(y)=\phi\left(y-x_{i}\right)$ converges uniformly in any compact set to a solution $u$ of

$$
-\Delta u-\left(2^{*}-1\right) U_{0, \Lambda}^{2^{*}-2} u=0, \quad \text { in } \mathbb{R}^{N},
$$

for some $\Lambda \in\left[L_{1}, L_{2}\right]$, and $u$ is perpendicular to the kernel of (2.13). Hence, $u=0$. This is a contradiction to (2.12).

From Lemma 2.1, using the same argument as in the proof of Proposition 4.1 in [5], we can prove the following result :

Proposition 2.2. There exists $k_{0}>0$ and a constant $C>0$, independent of $k$, such that for all $k \geq k_{0}$ and all $h \in L^{\infty}\left(\mathbb{R}^{N}\right)$, problem (2.3) has a unique solution $\phi \equiv L_{k}(h)$. Moreover,

$$
\left\|L_{k}(h)\right\|_{*} \leq C\|h\|_{* *}, \quad\left|c_{l}\right| \leq C\|h\|_{* *} .
$$

Now, we consider

$$
\left\{\begin{array}{l}
-\Delta\left(W_{r, \Lambda}+\phi\right)=K\left(\frac{y}{\mu}\right)\left(W_{r, \Lambda}+\phi\right)^{2^{*}-1}+\sum_{t=1}^{2} c_{t} \sum_{i=1}^{k} U_{x_{i}, \Lambda}^{2^{*}-2} Z_{i, t}, \quad \text { in } B_{\mu}(0), \\
\phi_{k} \in H_{s} \\
<U_{x_{i}, \Lambda}^{2^{*}-2} Z_{i, l}, \phi_{k}>=0, \quad i=1, \ldots, k, l=1,2 .
\end{array}\right.
$$

We have

Proposition 2.3. There is an integer $k_{0}>0$, such that for each $k \geq k_{0}, L_{0} \leq$ $\Lambda \leq L_{1}, r \in\left[\mu\left(1-r_{0} / k\right), \mu\left(1-r_{1} / k\right)\right]$, (2.15) has a unique solution $\phi=\phi(r, \Lambda)$, satisfying

$$
\|\phi\|_{*} \leq C\left(\frac{1}{\mu}\right)^{1 / 2+\sigma}, \quad\left|c_{t}\right| \leq C\left(\frac{1}{\mu}\right)^{1 / 2+\sigma},
$$

if $N \geq 4$, where $\sigma>0$ is a small constant, and $\mu=k^{\frac{N-1}{N-2}}$.

Rewrite (2.15) as

$$
\left\{\begin{array}{l}
-\Delta \phi-\left(2^{*}-1\right) K\left(\frac{|y|}{\mu}\right) W_{r, \Lambda}^{2^{*}-2} \phi=N(\phi)+l_{k}+\sum_{t=1}^{2} c_{i} \sum_{i=1}^{k} U_{x_{i}, \Lambda}^{2^{*}-2} Z_{i, t}, \text { in } B_{\mu}(0), \\
\phi \in H_{s} \\
<U_{x_{i}, \Lambda}^{2^{*}-2} Z_{i, l}, \phi>=0, \quad i=1, \ldots, k, l=1,2,
\end{array}\right.
$$


where

$$
N(\phi)=K\left(\frac{|y|}{\mu}\right)\left(\left(W_{r, \Lambda}+\phi\right)^{2^{*}-1}-W_{r, \Lambda}^{2^{*}-1}-\left(2^{*}-1\right) W_{r}^{2^{*}-2} \phi\right),
$$

and

$$
l_{k}=K\left(\frac{|y|}{\mu}\right) W_{r, \Lambda}^{2^{*}-1}-\sum_{j=1}^{k} U_{x_{j}, \Lambda}^{2^{*}-1} .
$$

In order to use the contraction mapping theorem to prove that $(2.16)$ is uniquely solvable in the set where $\|\phi\|_{*}$ is small, we need to estimate $N(\phi)$ and $l_{k}$.

Lemma 2.4. If $N \geq 4$, then

$$
\|N(\phi)\|_{* *} \leq C\|\phi\|_{*}^{\min \left(2^{*}-1,2\right)} .
$$

Proof. We have

$$
|N(\phi)| \leq \begin{cases}C|\phi|^{2^{*}-1}, & N \geq 6 \\ C\left(W_{r, \Lambda}^{\frac{6-N}{N-2}} \phi^{2}+|\phi|^{2^{*}-1}\right), & N=4,5 .\end{cases}
$$

First, we consider $N \geq 6$. Using

$$
\sum_{j=1}^{k} a_{j} b_{j} \leq\left(\sum_{j=1}^{k} a_{j}^{p}\right)^{1 / p}\left(\sum_{j=1}^{k} b_{j}^{q}\right)^{1 / q}, \quad \frac{1}{p}+\frac{1}{q}=1, a_{j}, b_{j} \geq 0,
$$

we obtain

$$
\begin{aligned}
|N(\phi)| & \leq C\|\phi\|_{*}^{2^{*}-1}\left(\sum_{j=1}^{k} \frac{1}{\left(1+\left|y-x_{j}\right|\right)^{\frac{N-2}{2}+\tau}}\right)^{2^{*}-1} \\
& \leq C\|\phi\|_{*}^{2^{*}-1} \sum_{j=1}^{k} \frac{1}{\left(1+\left|y-x_{j}\right|\right)^{\frac{N+2}{2}+\tau}}\left(\sum_{j=1}^{k} \frac{1}{\left(1+\left|y-x_{j}\right|\right)^{\tau}}\right)^{\frac{4}{N-2}} \\
& \leq C\|\phi\|_{*}^{2^{*}-1} \sum_{j=1}^{k} \frac{1}{\left(1+\left|y-x_{j}\right|\right)^{\frac{N+2}{2}+\tau}}
\end{aligned}
$$

Thus, the result follows.

Suppose that $N=4$ or 5 . Noting that $N-2 \geq(N-2) / 2+\tau$, we find

$$
\begin{aligned}
& |N(\phi)| \leq C\|\phi\|_{*}^{2}\left(\sum_{i=1}^{k} \frac{1}{\left(1+\left|y-x_{i}\right|\right)^{N-2}}\right)^{\frac{6-N}{N-2}}\left(\sum_{j=1}^{k} \frac{1}{\left.1+\left|y-x_{j}\right|\right)^{\frac{N-2}{2}+\tau}}\right)^{2} \\
& \quad+C\|\phi\|_{*}^{2^{*}-1} \sum_{j=1}^{k} \frac{1}{\left(1+\left|y-x_{j}\right|\right)^{\frac{N+2}{2}+\tau}} \\
& \leq C\|\phi\|_{*}^{2}\left(\sum_{j=1}^{k} \frac{1}{\left.1+\left|y-x_{j}\right|\right)^{\frac{N-2}{2}+\tau}}\right)^{2^{*}-1}+C\|\phi\|_{*}^{2^{*}-1} \sum_{j=1}^{k} \frac{1}{\left(1+\left|y-x_{j}\right|\right)^{\frac{N+2}{2}+\tau}} \\
& =C\|\phi\|_{*}^{2} \sum_{j=1}^{k} \frac{1}{\left(1+\left|y-x_{j}\right|\right)^{\frac{N+2}{2}+\tau}} .
\end{aligned}
$$


So, we have proved that, for $N \geq 4$,

$$
\|N(\phi)\|_{* *} \leq C\|\phi\|_{*}^{\min \left(2,2^{*}-1\right)} .
$$

Next, we estimate $l_{k}$.

Lemma 2.5. Assume that $r \in\left[\mu\left(1-r_{0} / k\right), \mu\left(1-r_{1} / k\right)\right]$. If $N \geq 4$, then

$$
\left\|l_{k}\right\|_{* *} \leq C\left(\frac{1}{\mu}\right)^{1 / 2+\sigma} .
$$

Proof. Define

$$
\Omega_{j}=\left\{y: y=\left(y^{\prime}, y^{\prime \prime}\right) \in B_{\mu}(0),\left\langle\frac{y^{\prime}}{\left|y^{\prime}\right|}, \frac{x_{j}}{\left|x_{j}\right|}\right\rangle \geq \cos \frac{\pi}{k}\right\} .
$$

We have

$$
\begin{aligned}
l_{k}= & K\left(\frac{|y|}{\mu}\right)\left(W_{r, \Lambda}^{2^{*}-1}-\sum_{j=1}^{k}\left(P U_{x_{j}, \Lambda}\right)^{2^{*}-1}\right) \\
& +K\left(\frac{|y|}{\mu}\right)\left(\sum_{j=1}^{k}\left(P U_{x_{j}, \Lambda}\right)^{2^{*}-1}-\sum_{j=1}^{k} U_{x_{j}, \Lambda}^{2^{*}-1}\right)+\sum_{j=1}^{k} U_{x_{j}, \Lambda}^{2^{*}-1}\left(K\left(\frac{|y|}{\mu}\right)-1\right) \\
= & : J_{0}+J_{1}+J_{2} .
\end{aligned}
$$

Using the assumed symmetry, we can suppose that $y \in \Omega_{1}$. Then,

$$
\left|y-x_{j}\right| \geq\left|y-x_{1}\right|, \quad \forall y \in \Omega_{1} \text {. }
$$

First, we claim

$$
\frac{1}{1+\left|y-x_{j}\right|} \leq \frac{C}{\left|x_{j}-x_{1}\right|}, \quad \forall y \in \Omega_{1}, j \neq 1
$$

In fact, if $\left|y-x_{1}\right| \leq \frac{1}{2}\left|x_{1}-x_{j}\right|$, then $\left|y-x_{j}\right| \geq \frac{1}{2}\left|x_{1}-x_{j}\right|$. If $\left|y-x_{1}\right| \geq \frac{1}{2}\left|x_{1}-x_{j}\right|$, then $\left|y-x_{j}\right| \geq\left|y-x_{1}\right| \geq \frac{1}{2}\left|x_{1}-x_{j}\right|$, since $y \in \Omega_{1}$.

For the estimate of $J_{0}$, we have

$$
\begin{aligned}
\left|J_{0}\right| \leq & C \frac{1}{\left(1+\left|y-x_{1}\right|\right)^{4}} \sum_{j=2}^{k} \frac{1}{\left(1+\left|y-x_{j}\right|\right)^{N-2}} \\
& +C\left(\sum_{j=2}^{k} \frac{1}{\left(1+\left|y-x_{j}\right|\right)^{N-2}}\right)^{2^{*}-1} .
\end{aligned}
$$

Using (2.18), and taking $1<\alpha \leq N-2$, we obtain that, for any $y \in \Omega_{1}$,

$$
\begin{aligned}
& \frac{1}{\left(1+\left|y-x_{1}\right|\right)^{4}} \frac{1}{\left(1+\left|y-x_{j}\right|\right)^{N-2}} \\
& \quad \leq C \frac{1}{\left(1+\left|y-x_{1}\right|\right)^{N+2-\alpha}} \frac{1}{\left|x_{j}-x_{1}\right|^{\alpha}}, \quad j>1 .
\end{aligned}
$$


Take $\alpha>\max ((N-1) / 2,1)$ satisfying $N+2-\alpha \geq(N+2) / 2+\tau$. Then

$$
\begin{gathered}
\frac{1}{\left(1+\left|y-x_{1}\right|\right)^{4}} \sum_{j=2}^{k} \frac{1}{\left(1+\left|y-x_{j}\right|\right)^{N-2}} \leq \frac{C}{\left(1+\left|y-x_{1}\right|\right)^{N+2-\alpha}}\left(\frac{k}{\mu}\right)^{\alpha} \\
=\frac{C}{\left(1+\left|y-x_{1}\right|\right)^{N+2-\alpha}} \mu^{-\alpha /(N-1)} \leq C \frac{1}{\left(1+\left|y-x_{1}\right|\right)^{\frac{N+2}{2}+\tau}}\left(\frac{1}{\mu}\right)^{1 / 2+\sigma} .
\end{gathered}
$$

Using the Hölder inequality, we obtain

$$
\begin{aligned}
& \left(\sum_{j=2}^{k} \frac{1}{\left(1+\left|y-x_{j}\right|\right)^{N-2}}\right)^{2^{*}-1} \\
& \quad \leq \sum_{j=2}^{k} \frac{1}{\left(1+\left|y-x_{j}\right|\right)^{\frac{N+2}{2}+\tau}}\left(\sum_{j=2}^{k} \frac{1}{\left(1+\left|y-x_{j}\right|\right)^{\frac{N+2}{4}\left(\frac{N-2}{2}-\tau \frac{N-2}{N+2}\right)}}\right)^{4 /(N-2)} .
\end{aligned}
$$

Noting that $\frac{N+2}{4}\left(\frac{N-2}{2}-\tau \frac{N-2}{N+2}\right)>1$ if $N \geq 4$, we obtain

$$
\begin{aligned}
& \left(\sum_{j=2}^{k} \frac{1}{\left(1+\left|y-x_{j}\right|\right)^{N-2}}\right)^{2^{*}-1} \\
& \quad \leq C\left(\sum_{j=2}^{k} \frac{1}{\left|x_{1}-x_{j}\right|^{\frac{N+2}{4}\left(\frac{N-2}{2}-\tau \frac{N-2}{N+2}\right)}}\right)^{4 /(N-2)} \sum_{j=1}^{k} \frac{1}{\left(1+\left|y-x_{j}\right|\right)^{\frac{N+2}{2}+\tau}} \\
& \quad \leq C\left(\frac{k}{\mu}\right)^{\frac{N+2}{4}\left(\frac{N-2}{2}-\tau \frac{N-2}{N+2}\right) \frac{4}{N-2}} \sum_{j=1}^{k} \frac{1}{\left(1+\left|y-x_{j}\right|\right)^{\frac{N+2}{2}+\tau}} \\
& \quad=C\left(\frac{1}{\mu}\right)^{\frac{N+2}{N-1}\left(\frac{1}{2}-\frac{\tau}{N+2}\right)} \sum_{j=1}^{k} \frac{1}{\left(1+\left|y-x_{j}\right|\right)^{\frac{N+2}{2}+\tau}} \\
& \quad=C\left(\frac{1}{\mu}\right)^{1 / 2+\sigma} \sum_{j=1}^{k} \frac{1}{\left(1+\left|y-x_{j}\right|\right)^{\frac{N+2}{2}+\tau}},
\end{aligned}
$$

since $\frac{N+2}{N-1}\left(\frac{1}{2}-\frac{\tau}{N+2}\right)>\frac{1}{2}$. Thus, we have proved that if $N \geq 4$,

$$
\left\|J_{0}\right\|_{* *} \leq C\left(\frac{1}{\mu}\right)^{1 / 2+\sigma}
$$

Now, we estimate $J_{1}$. Let $H(y, x)$ be the regular part of the Green function for $-\Delta$ in $B_{1}(0)$ with the zero boundary condition. Let $\bar{x}_{j}^{*}$ be the reflection point of $\bar{x}_{j}$ with respect to $\partial B_{1}(0)$. Then

$$
\frac{H\left(\bar{y}, \bar{x}_{j}\right)}{\mu^{N-2}}=\frac{C}{\mu^{N-2}\left|\bar{y}-\bar{x}_{j}^{*}\right|^{N-2}} \leq \frac{C}{\left(1+\left|y-x_{j}\right|\right)^{N-2}} .
$$


Take $t=1-\theta$ with $\theta>0$ small. Then using (A.1), we find

$$
\begin{aligned}
\left|J_{1}\right| & \leq \sum_{j=1}^{k} \frac{C}{\left(1+\left|y-x_{j}\right|\right)^{4}} \frac{H\left(\bar{y}, \bar{x}_{j}\right)}{\mu^{N-2}} \\
& \leq \sum_{j=1}^{k} \frac{C}{\left(1+\left|y-x_{j}\right|\right)^{4+t(N-2)}}\left(\frac{H\left(\bar{y}, \bar{x}_{j}\right)}{\mu^{N-2}}\right)^{t} \\
& \leq C\left(\frac{1}{\mu d}\right)^{t(N-2)} \sum_{j=1}^{k} \frac{1}{\left(1+\left|y-x_{j}\right|\right)^{4+t(N-2)}} \\
& \leq C\left(\frac{1}{\mu}\right)^{t \frac{N-2}{N-1}} \sum_{j=1}^{k} \frac{1}{\left(1+\left|y-x_{j}\right|\right)^{4+t(N-2)}} \\
& \leq C\left(\frac{1}{\mu}\right)^{1 / 2+\sigma} \sum_{j=1}^{k} \frac{1}{\left(1+\left|y-x_{j}\right|\right)^{\frac{N+2}{2}+\tau}},
\end{aligned}
$$

since $t \frac{N-2}{N-1}>1 / 2$ for $N \geq 4,4+t(N-2) \geq(N+2) / 2+\tau$, and $d \geq r_{0} / k$.

Finally, we estimate $J_{2}$. For $y \in \Omega_{1}$, and $j>1$, using (2.18), we have

$$
U_{x_{j}, \Lambda}^{2^{*}-1}(y) \leq C \frac{1}{\left(1+\left|y-x_{1}\right|\right)^{\frac{N+2}{2}+\tau}} \frac{1}{\left|x_{1}-x_{j}\right|^{\frac{N+2}{2}-\tau}},
$$

which implies

$$
\begin{aligned}
& \left|\sum_{j=2}^{k}\left(K\left(\frac{|y|}{\mu}\right)-1\right) U_{x_{j}, \Lambda}^{2^{*}-1}\right| \leq C \frac{1}{\left(1+\left|y-x_{1}\right|\right)^{\frac{N+2}{2}+\tau}} \sum_{j=2}^{k} \frac{1}{\left|x_{1}-x_{j}\right|^{\frac{N+2}{2}-\tau}} \\
& 24) \quad \leq C \frac{1}{\left(1+\left|y-x_{1}\right|\right)^{\frac{N+2}{2}+\tau}}\left(\frac{k}{\mu}\right)^{\frac{N+2}{2}-\tau} \leq C \frac{1}{\left(1+\left|y-x_{1}\right|\right)^{\frac{N+2}{2}+\tau}}\left(\frac{1}{\mu}\right)^{1 / 2+\sigma} .
\end{aligned}
$$

For $y \in \Omega_{1}$ and ||$y|-\mu| \geq \delta \mu$, where $\delta>0$ is a fixed constant, then

$$
|| y|-| x_{1}|| \geq|| y|-\mu|-|| x_{1}|-\mu| \geq \frac{1}{2} \delta \mu \text {. }
$$

As a result,

$$
\left|U_{x_{1}, \Lambda}^{2^{*}-1}\left(K\left(\frac{|y|}{\mu}\right)-1\right)\right| \leq C \frac{1}{\left(1+\left|y-x_{1}\right|\right)^{\frac{N+2}{2}+\tau}} \frac{1}{\mu^{\frac{N+2}{2}-\tau}} .
$$

If $y \in \Omega_{1}$ and ||$y|-\mu| \leq \delta \mu$, then

$$
\begin{aligned}
\left|K\left(\frac{|y|}{\mu}\right)-1\right| \leq C\left|\frac{|y|}{\mu}-1\right| & \left.\leq \frac{C}{\mu}\left(\left(|| y|-| x_{1}||\right)+|| x_{1}|-\mu|\right)\right) \\
\leq \frac{C}{\mu}|| y|-| x_{1}||+\frac{C}{k} & =\frac{C}{\mu}|| y|-| x_{1}||+\frac{C}{\mu^{\frac{N-2}{N-1}}} \leq \frac{C}{\mu}|| y|-| x_{1}||+\frac{C}{\mu^{1 / 2+\sigma}},
\end{aligned}
$$

and

$$
|| y|-| x_{1}|| \leq|| y|-\mu|+|\mu-| x_{1}|| \leq 2 \delta \mu .
$$


However,

$$
\begin{aligned}
& \frac{\| y|-| x_{1}||}{\mu} \frac{1}{\left(1+\left|y-x_{1}\right|\right)^{N+2}}=\frac{C}{\mu^{1 / 2+\sigma}} \frac{\| y|-| x_{1}||^{1 / 2-\sigma}}{\left(1+\left|y-x_{1}\right|\right)^{N+2}} \\
& \quad \leq \frac{C}{\mu^{1 / 2+\sigma}} \frac{1}{\left(1+\left|y-x_{1}\right|\right)^{N+2-1 / 2+\sigma}} \leq \frac{C}{\mu^{1 / 2+\sigma}} \frac{1}{\left(1+\left|y-x_{1}\right|\right)^{\frac{N+2}{2}+\tau}} .
\end{aligned}
$$

Thus, we obtain

$$
\left|U_{x_{1}, \Lambda}^{2^{*}-1}\left(K\left(\frac{|y|}{\mu}\right)-1\right)\right| \leq \frac{C}{\mu^{1 / 2+\sigma}} \frac{1}{\left(1+\left|y-x_{1}\right|\right)^{\frac{N+2}{2}+\tau}}, \quad|| y|-\mu| \leq \delta \mu .
$$

Combining (2.24), (2.25) and (2.26), we obtain

$$
\left\|J_{2}\right\|_{* *} \leq C\left(\frac{1}{\mu}\right)^{1 / 2+\sigma}
$$

Now, we are ready to prove Proposition 2.3.

Proof of Proposition 2.3. Let us recall that

$$
\mu=k^{\frac{N-1}{N-2}}, \quad N \geq 4 .
$$

Let

$$
\begin{aligned}
E=\left\{u: u \in C\left(B_{\mu}(0)\right) \cap H_{s},\|u\|_{*} \leq\left(\frac{1}{k}\right)^{1 / 2},\right. & \\
& \left.\int_{B_{\mu}(0)} U_{x_{i}, \Lambda}^{2 *-2} Z_{i, l} \phi=0, i=1, \ldots, k, l=1,2\right\} .
\end{aligned}
$$

Then, (2.16) is equivalent to

$$
\phi=A(\phi)=: L_{k}(N(\phi))+L_{k}\left(l_{k}\right),
$$

where $L_{k}$ is defined in Proposition 2.2. We will prove that $A$ is a contraction map from $E$ to $E$.

We have

$$
\begin{aligned}
\|A(\phi)\|_{*} & \leq C\|N(\phi)\|_{*_{*}}+C\left\|l_{k}\right\|_{* *} \\
& \leq C\|\phi\|_{*}^{\min \left(2^{*}-1,2\right)}+C\left\|l_{k}\right\|_{* *} \leq \frac{C}{k^{1 / 2+\sigma}} \leq \frac{1}{k^{1 / 2}} .
\end{aligned}
$$

Thus, $A$ maps $E$ to $E$.

On the other hand,

$$
\left\|A\left(\phi_{1}\right)-A\left(\phi_{2}\right)\right\|_{*}=\left\|L_{k}\left(N\left(\phi_{1}\right)\right)-L_{k}\left(N\left(\phi_{2}\right)\right)\right\|_{*} \leq C\left\|N\left(\phi_{1}\right)-N\left(\phi_{2}\right)\right\|_{* *} .
$$

If $N \geq 6$, then

$$
\left|N^{\prime}(t)\right| \leq C|t|^{2^{*}-2}
$$


As a result,

$$
\begin{aligned}
& \left|N\left(\phi_{1}\right)-N\left(\phi_{2}\right)\right| \leq C\left(\left|\phi_{1}\right|^{2^{*}-2}+\left|\phi_{2}\right|^{2^{*}-2}\right)\left|\phi_{1}-\phi_{2}\right| \\
& \quad \leq C\left(\left\|\phi_{1}\right\|_{*}^{2^{*}-2}+\left\|\phi_{2}\right\|_{*}^{2^{*}-2}\right)\left\|\phi_{1}-\phi_{2}\right\|_{*}\left(\sum_{j=1}^{k} \frac{1}{\left(1+\left|y-x_{j}\right|\right)^{\frac{N-2}{2}+\tau}}\right)^{2^{*}-1} .
\end{aligned}
$$

As before, we have

$$
\left(\sum_{j=1}^{k} \frac{1}{\left(1+\left|y-x_{j}\right|\right)^{\frac{N-2}{2}+\tau}}\right)^{2^{*}-1} \leq C \sum_{j=1}^{k} \frac{1}{\left(1+\left|y-x_{j}\right|\right)^{\frac{N+2}{2}+\tau}}
$$

So,

$$
\begin{aligned}
\left\|A\left(\phi_{1}\right)-A\left(\phi_{2}\right)\right\|_{*} & \leq C\left\|N\left(\phi_{1}\right)-N\left(\phi_{2}\right)\right\|_{* *} \\
& \leq C\left(\left\|\phi_{1}\right\|_{*}^{2^{*}-2}+\left\|\phi_{2}\right\|_{*}^{2^{*}-2}\right)\left\|\phi_{1}-\phi_{2}\right\|_{*} \leq \frac{1}{2}\left\|\phi_{1}-\phi_{2}\right\|_{*} .
\end{aligned}
$$

Thus, $A$ is a contraction map.

For $N=4$ or 5 ,

$$
\left|N^{\prime}(t)\right| \leq C W_{r, \Lambda}^{\frac{6-N}{N-2}}|t|+C|t|^{2^{*}-2} .
$$

So,

$$
\begin{aligned}
& \left|N\left(\phi_{1}\right)-N\left(\phi_{2}\right)\right| \\
& \leq C\left(\left|\phi_{1}\right|^{2^{*}-2}+\left|\phi_{2}\right|^{2^{*}-2}\right)\left|\phi_{1}-\phi_{2}\right|+C\left(\left|\phi_{1}\right|+\left|\phi_{2}\right|\right) W_{r, \Lambda}^{\frac{6-N}{N-2}}\left|\phi_{1}-\phi_{2}\right| \\
& \leq C\left(\left\|\phi_{1}\right\|_{*}^{2^{*}-2}+\left\|\phi_{2}\right\|_{*}^{2^{*}-2}\right)\left\|\phi_{1}-\phi_{2}\right\|_{*}\left(\sum_{j=1}^{k} \frac{1}{\left(1+\left|y-x_{j}\right|\right)^{\frac{N-2}{2}+\tau}}\right)^{2^{*}-1} \\
& \quad+C\left(\left\|\phi_{1}\right\|_{*}+\left\|\phi_{2}\right\|_{*}\right)\left\|\phi_{1}-\phi_{2}\right\|_{*} W_{r, \Lambda}^{\frac{6-N}{N-2}}\left(\sum_{j=1}^{k} \frac{1}{\left(1+\left|y-x_{j}\right|\right)^{\frac{N-2}{2}+\tau}}\right)^{2} \\
& \quad \leq C\left(\left\|\phi_{1}\right\|_{*}+\left\|\phi_{2}\right\|_{*}\right)\left\|\phi_{1}-\phi_{2}\right\|_{*} \sum_{j=1}^{k} \frac{1}{\left(1+\left|y-x_{j}\right|\right)^{\frac{N+2}{2}+\tau}} .
\end{aligned}
$$

Thus, $A$ is a contraction map.

It follows from the contraction mapping theorem that there is a unique $\phi \in E$, such that

$$
\phi=A(\phi) .
$$

Moreover, it follows from Proposition 2.2 that

$$
\|\phi\|_{*} \leq C\left\|l_{k}\right\|_{* *}+C\|N(\phi)\|_{* *} \leq C\left\|l_{k}\right\|_{* *}+C\|\phi\|_{*}^{\min \left(2^{*}-1,2\right)},
$$

which gives, if $N \geq 4$,

$$
\|\phi\|_{*} \leq C\left(\frac{1}{\mu}\right)^{1 / 2+\sigma}
$$

Finally, the estimate of $c_{t}$ comes from (2.14). See also (2.10). 


\section{Proof of Theorem 1.2}

Let

$$
F(d, \Lambda)=I\left(W_{r, \Lambda}+\phi\right)
$$

where $r=\left|x_{1}\right|, d=1-r / \mu, \phi$ is the function obtained in Proposition 2.3, and

$$
I(u)=\frac{1}{2} \int_{B_{\mu}(0)}|D u|^{2}-\frac{1}{2^{*}} \int_{B_{\mu}(0)} K\left(\frac{|y|}{\mu}\right)|u|^{2^{*}} .
$$

Proposition 3.1. If $N \geq 4$, then

$$
\begin{aligned}
F(d, \Lambda) & =I\left(W_{r, \Lambda}\right)+O\left(\frac{1}{\mu^{1+\sigma}}\right) \\
& =k\left(A+\frac{B_{1} H\left(\bar{x}_{1}, \bar{x}_{1}\right)}{\Lambda^{N-2} \mu^{N-2}}+B_{2} K^{\prime}(1) d-\sum_{i=2}^{k} \frac{B_{1} G\left(\bar{x}_{i}, \bar{x}_{1}\right)}{\Lambda^{N-2} \mu^{N-2}}+O\left(\frac{1}{\mu^{1+\sigma}}\right)\right),
\end{aligned}
$$

where $A, B_{1}$ and $B_{2}$ are positive constants, and $\sigma>0$ is a small constant.

Proof. Since

$$
\left\langle I^{\prime}\left(W_{r, \Lambda}+\phi\right), \phi\right\rangle=0, \quad \forall \phi \in E,
$$

there is $t \in(0,1)$ such that

$$
\begin{aligned}
F(d, \Lambda)= & I\left(W_{r, \Lambda}\right)-\frac{1}{2} D^{2} I\left(W_{r, \Lambda}+t \phi\right)(\phi, \phi) \\
= & I\left(W_{r, \Lambda}\right)-\frac{1}{2} \int_{B_{\mu}(0)}\left(|D \phi|^{2}-\left(2^{*}-1\right) K\left(\frac{|y|}{\mu}\right)\left(W_{r, \Lambda}+t \phi\right)^{2^{*}-2} \phi^{2}\right) \\
= & I\left(W_{r, \Lambda}\right)+\frac{2^{*}-1}{2} \int_{B_{\mu}(0)} K\left(\frac{|y|}{\mu}\right)\left(\left(W_{r, \Lambda}+t \phi\right)^{2^{*}-2}-W_{r, \Lambda}^{2^{*}-2}\right) \phi^{2} \\
& -\frac{1}{2} \int_{B_{\mu}(0)}\left(N(\phi)+l_{k}\right) \phi \\
= & I\left(W_{r, \Lambda}\right)+O\left(\int_{B_{\mu}(0)}\left(|\phi|^{2^{*}}+|N(\phi)||\phi|+\left|l_{k}\right||\phi|\right)\right) .
\end{aligned}
$$

However,

$$
\begin{aligned}
\int_{B_{\mu}(0)}\left(|N(\phi)||\phi|+\left|l_{k}\right||\phi|\right) \leq C\left(\|N(\phi)\|_{* *}+\left\|l_{k}\right\|_{* *}\right)\|\phi\|_{*} \\
\quad \times \int_{B_{\mu}(0)} \sum_{j=1}^{k} \frac{1}{\left(1+\left|y-x_{j}\right|\right)^{\frac{N+2}{2}+\tau}} \sum_{i=1}^{k} \frac{1}{\left(1+\left|y-x_{i}\right|\right)^{\frac{N-2}{2}+\tau}} .
\end{aligned}
$$


Using Lemma B.1, for $N \geq 4$,

$$
\begin{aligned}
& \sum_{j=1}^{k} \frac{1}{\left(1+\left|y-x_{j}\right|\right)^{\frac{N+2}{2}+\tau}} \sum_{i=1}^{k} \frac{1}{\left(1+\left|y-x_{i}\right|\right)^{\frac{N-2}{2}+\tau}} \\
& \quad=\sum_{j=1}^{k} \frac{1}{\left(1+\left|y-x_{j}\right|\right)^{N+2 \tau}}+\sum_{j=1}^{k} \sum_{i \neq j} \frac{1}{\left(1+\left|y-x_{j}\right|\right)^{\frac{N+2}{2}+\tau}} \frac{1}{\left(1+\left|y-x_{i}\right|\right)^{\frac{N-2}{2}+\tau}} \\
& \quad \leq \sum_{j=1}^{k} \frac{1}{\left(1+\left|y-x_{j}\right|\right)^{N+2 \tau}}+C \sum_{j=1}^{k} \frac{1}{\left(1+\left|y-x_{j}\right|\right)^{N+\tau}} \sum_{j=2}^{k} \frac{1}{\left|x_{j}-x_{1}\right|^{\tau}} \\
& \quad \leq C \sum_{j=1}^{k} \frac{1}{\left(1+\left|y-x_{j}\right|\right)^{N+\tau}} .
\end{aligned}
$$

Thus, we obtain

$$
\int_{B_{\mu}(0)}\left(\left|N(\phi)\left\|\phi|+| l_{k}\right\| \phi\right|\right) \leq C k\left(\|N(\phi)\|_{* *}+\left\|l_{k}\right\|_{* *}\right)\|\phi\|_{*} \leq C k\left(\frac{1}{\mu}\right)^{1+\sigma}, \quad N \geq 4 .
$$

On the other hand,

$$
\int_{B_{\mu}(0)}|\phi|^{2^{*}} \leq C\|\phi\|_{*}^{2^{*}} \int_{B_{\mu}(0)}\left(\sum_{j=1}^{k} \frac{1}{\left(1+\left|y-x_{j}\right|\right)^{\frac{N-2}{2}+\tau}}\right)^{2^{*}} .
$$

However, using (2.18), if $y \in \Omega_{1}$, and $N \geq 4$,

$$
\begin{aligned}
& \sum_{j=2}^{k} \frac{1}{\left(1+\left|y-x_{j}\right|\right)^{\frac{N-2}{2}+\tau}} \\
& \quad \leq C \frac{1}{\left(1+\left|y-x_{1}\right|\right)^{\frac{N-2}{2}}} \sum_{j=2}^{k} \frac{1}{\left|x_{j}-x_{1}\right|^{\tau}} \leq C \frac{1}{\left(1+\left|y-x_{1}\right|\right)^{\frac{N-2}{2}}}
\end{aligned}
$$

Thus,

$$
\left(\sum_{j=1}^{k} \frac{1}{\left(1+\left|y-x_{j}\right|\right)^{\frac{N-2}{2}+\tau}}\right)^{2^{*}} \leq \frac{C}{\left(1+\left|y-x_{1}\right|\right)^{N}}, \quad y \in \Omega_{1},
$$

which gives

$$
\int_{B_{\mu}(0)}\left(\sum_{j=1}^{k} \frac{1}{\left(1+\left|y-x_{j}\right|\right)^{\frac{N-2}{2}+\tau}}\right)^{2^{*}} \leq C k \ln k .
$$

So, we have proved

$$
\int_{B_{\mu}(0)}|\phi|^{2^{*}} \leq C k \ln k\|\phi\|_{*}^{2^{*}} \leq C k \ln k\left(\frac{1}{\mu}\right)^{2^{*}(1 / 2+\sigma)}, \quad N \geq 4 .
$$


Proposition 3.2. We have

$$
\frac{\partial F(d, \Lambda)}{\partial \Lambda}=k B_{1}(N-2)\left(-\frac{H\left(\bar{x}_{1}, \bar{x}_{1}\right)}{\Lambda^{N-1} \mu^{N-2}}+\sum_{i=2}^{k} \frac{G\left(\bar{x}_{i}, \bar{x}_{1}\right)}{\Lambda^{N-1} \mu^{N-2}}+O\left(\frac{1}{\mu^{1+\sigma}}\right)\right)
$$

and

$$
\frac{\partial F(d, \Lambda)}{\partial d}=k\left(\frac{B_{1} \frac{\partial H\left(\bar{x}_{1}, \bar{x}_{1}\right)}{\partial d}}{\Lambda^{N-2} \mu^{N-2}}+B_{2} K^{\prime}(1)-\sum_{i=2}^{k} \frac{B_{1} \frac{\partial G\left(\bar{x}_{i}, \bar{x}_{1}\right)}{\partial d}}{\Lambda^{N-1} \mu^{N-2}}+O\left(\frac{1}{\mu^{\sigma}}\right)\right)
$$

if $N \geq 4$, where $B_{1}$ and $B_{2}$ are the same constants as in Proposition 3.1, and $\sigma>0$ is a small constant.

Proof. We estimate $\partial F(d, \Lambda) / \partial \Lambda$ first. We have

$$
\begin{aligned}
\frac{\partial F(d, \Lambda)}{\partial \Lambda} & =\left\langle I^{\prime}\left(W_{r, \Lambda}+\phi\right), \frac{\partial W_{r, \Lambda}}{\partial \Lambda}+\frac{\partial \phi}{\partial \Lambda}\right\rangle \\
& =\left\langle I^{\prime}\left(W_{r, \Lambda}+\phi\right), \frac{\partial W_{r, \Lambda}}{\partial \Lambda}\right\rangle+\sum_{l=1}^{2} \sum_{i=1}^{k} c_{l}\left\langle U_{x_{i}, \Lambda}^{2^{*}-2} Z_{i, l}, \frac{\partial \phi}{\partial \Lambda}\right\rangle
\end{aligned}
$$

However,

$$
\left\langle U_{x_{i}, \Lambda}^{2^{*}-2} Z_{i, l}, \frac{\partial \phi}{\partial \Lambda}\right\rangle=-\left\langle\frac{\partial\left(U_{x_{i}, \Lambda}^{2^{*}-2} Z_{i, l}\right)}{\partial \Lambda}, \phi\right\rangle
$$

Thus, using Proposition 2.3,

$$
\begin{aligned}
& \left|\sum_{i=1}^{k} c_{l}\left\langle U_{x_{i}, \Lambda}^{2^{*}-2} Z_{i, l}, \frac{\partial \phi}{\partial \Lambda}\right\rangle\right| \\
& \quad \leq C\left|c_{l}\right|\|\phi\|_{*} \int_{\mathbb{R}^{N}} \sum_{i=1}^{k} \frac{1}{\left(1+\left|y-x_{i}\right|\right)^{N+2}} \sum_{j=1}^{k} \frac{1}{\left(1+\left|y-x_{j}\right|\right)^{\frac{N-2}{2}+\tau}} \leq \frac{C}{\mu^{1+\sigma}} .
\end{aligned}
$$

On the other hand,

$$
\int_{\mathbb{R}^{N}} D\left(W_{r, \Lambda}+\phi\right) D \frac{\partial W_{r, \Lambda}}{\partial \Lambda}=\int_{\mathbb{R}^{N}} D W_{r, \Lambda} D \frac{\partial W_{r, \Lambda}}{\partial \Lambda},
$$

and

$$
\begin{aligned}
& \int_{\mathbb{R}^{N}} K\left(\frac{|y|}{\mu}\right)\left(W_{r, \Lambda}+\phi\right)^{2^{*}-1} \frac{\partial W_{r, \Lambda}}{\partial \Lambda} \\
& \quad=\int_{\mathbb{R}^{N}} K\left(\frac{|y|}{\mu}\right) W_{r, \Lambda}^{2^{*}-1} \frac{\partial W_{r, \Lambda}}{\partial \Lambda}+\left(2^{*}-1\right) \int_{\mathbb{R}^{N}} K\left(\frac{|y|}{\mu}\right) W_{r, \Lambda}^{2^{*}-2} \frac{\partial W_{r, \Lambda}}{\partial \Lambda} \phi+O\left(\int_{\mathbb{R}^{N}}|\phi|^{2^{*}}\right) .
\end{aligned}
$$


Moreover, from $\phi \in E$,

$$
\begin{gathered}
\int_{\mathbb{R}^{N}} K\left(\frac{|y|}{\mu}\right) W_{r, \Lambda}^{2^{*}-2} \frac{\partial W_{r, \Lambda}}{\partial \Lambda} \phi \\
=\int_{\mathbb{R}^{N}} K\left(\frac{|y|}{\mu}\right)\left(W_{r, \Lambda}^{2^{*}-2} \frac{\partial W_{r, \Lambda}}{\partial \Lambda}-\sum_{j=1}^{k} U_{x_{j}, \Lambda}^{2^{*}-2} \frac{\partial U_{x_{j}, \Lambda}}{\partial \Lambda}\right) \phi \\
+\sum_{j=1}^{k} \int_{\mathbb{R}^{N}}\left(K\left(\frac{|y|}{\mu}\right)-1\right) U_{x_{j}, \Lambda}^{2^{*}-2} \frac{\partial U_{x_{j}, \Lambda}}{\partial \Lambda} \phi \\
=k \int_{\Omega_{1}} K\left(\frac{|y|}{\mu}\right)\left(W_{r, \Lambda}^{2^{*}-2} \frac{\partial W_{r, \Lambda}}{\partial \Lambda}-\sum_{j=1}^{k} U_{x_{j}, \Lambda}^{2^{*}-2} \frac{\partial U_{x_{j}, \Lambda}}{\partial \Lambda}\right) \phi \\
+k \int_{\mathbb{R}^{N}}\left(K\left(\frac{|y|}{\mu}\right)-1\right) U_{x_{1}, \Lambda}^{2^{*}-2} \frac{\partial U_{x_{1}, \Lambda}}{\partial \Lambda} \phi, \\
\left|\int_{\Omega_{1}} K\left(\frac{|y|}{\mu}\right)\left(W_{r, \Lambda}^{2^{*}-2} \frac{\partial W_{r, \Lambda}}{\partial \Lambda}-\sum_{j=1}^{k} U_{x_{j}, \Lambda}^{2^{*}-2} \frac{\partial U_{x_{j}, \Lambda}}{\partial \Lambda}\right) \phi\right| \\
\leq C \int_{\Omega_{1}}\left(U_{x_{1}, \Lambda}^{2^{*}-2}\left(U_{x_{1}, \Lambda}-P U_{x_{1}, \Lambda}\right)+U_{x_{1}, \Lambda}^{2^{*}-2} \sum_{j=2}^{k} U_{x_{j}, \Lambda}+\sum_{j=2}^{k} U_{x_{j}, \Lambda}^{2^{*}-1}\right)|\phi| \leq \frac{C}{\mu^{1+\sigma}}
\end{gathered}
$$

and

$$
\begin{aligned}
\left|\int_{\mathbb{R}^{N}}\left(K\left(\frac{|y|}{\mu}\right)-1\right) U_{x_{1}, \Lambda}^{2^{*}-2} \frac{\partial U_{x_{1}, \Lambda}}{\partial \Lambda} \phi\right| & \left|\int_{|| y|-\mu| \leq \sqrt{\mu}}\left(K\left(\frac{|y|}{\mu}\right)-1\right) U_{x_{1}, \Lambda}^{2^{*}-2} \frac{\partial U_{x_{1}, \Lambda}}{\partial \Lambda} \phi\right| \\
& +\left|\int_{|| y|-\mu| \geq \sqrt{\mu}}\left(K\left(\frac{|y|}{\mu}\right)-1\right) U_{x_{1}, \Lambda}^{2^{*}-2} \frac{\partial U_{x_{1}, \Lambda}}{\partial \Lambda} \phi\right| \\
\leq & \frac{C}{\mu^{1+\sigma}} .
\end{aligned}
$$

Thus, we have proved

$$
\frac{\partial F(d, \Lambda)}{\partial \Lambda}=\frac{\partial I\left(W_{r, \Lambda}\right)}{\partial \Lambda}+O\left(\frac{1}{\mu^{1+\sigma}}\right)
$$

and the result follows from Proposition A.2.

Finally, noting that $\partial / \partial d=-\mu \partial / \partial r$, we can estimate $\partial F(d, \Lambda) / \partial d$ in a similar way.

Now, we estimate $H\left(\bar{x}_{1}, \bar{x}_{1}\right)$ and $G\left(\bar{x}_{i}, \bar{x}_{1}\right), i \geq 2$. Let $\bar{x}_{1}^{*}=(1 /(1-d), 0, \ldots, 0)$ be the reflection of $\bar{x}_{1}$ with respect to the unit sphere. Then

$$
H\left(y, \bar{x}_{1}\right)=\frac{1}{\left|y-\bar{x}_{1}^{*}\right|^{N-2}}(1+O(d)) .
$$


So, we obtain

$$
H\left(\bar{x}_{1}, \bar{x}_{1}\right)=\frac{1}{2^{N-2} d^{N-2}}(1+O(d)) .
$$

On the other hand,

$$
\left|\bar{x}_{i}-\bar{x}_{1}^{*}\right|=\sqrt{\left|\bar{x}_{i}-\bar{x}_{1}\right|^{2}+4 d^{2}-4 d\left|\bar{x}_{i}-\bar{x}_{1}\right| \cos \theta_{i}},
$$

where $\theta_{i}$ is the angle between $\bar{x}_{i}-\bar{x}_{1}$ and $(1,0, \ldots, 0)$. Thus, $\theta_{i}=\pi / 2+(i-1) \pi / 2$.

$$
\begin{aligned}
G\left(\bar{x}_{i}, \bar{x}_{1}\right) & =\frac{1}{\left|\bar{x}_{i}-\bar{x}_{1}\right|^{N-2}}-\frac{1}{\left|\bar{x}_{i}-\bar{x}_{1}^{*}\right|^{N-2}}(1+O(d)) \\
& =\frac{1}{\left|\bar{x}_{i}-\bar{x}_{1}\right|^{N-2}}\left(1-\frac{1+O(d)}{\left(1+\frac{4 d^{2}+4 d\left|\bar{x}_{i}-\bar{x}_{1}\right| \sin ((i-1) \pi / 2)}{\left|\bar{x}_{i}-\bar{x}_{1}\right|^{2}}\right)^{(N-2) / 2}}\right) .
\end{aligned}
$$

Since

$$
\left|\bar{x}_{i}-x_{1}\right|=2\left|x_{1}\right| \sin \frac{(i-1) \pi}{k}, \quad i=2, \ldots, k,
$$

using $d k \rightarrow c>0$ and

$$
0<c^{\prime} \leq \frac{\sin ((j-1) \pi / k)}{(j-1) \pi / k} \leq c^{\prime \prime}, \quad j=2, \ldots,[k / 2]
$$

we obtain

$$
\frac{a_{0}}{j^{2}} \leq \frac{4 d^{2}+4 d\left|\bar{x}_{i}-\bar{x}_{1}\right| \sin ((i-1) \pi / 2)}{\left|\bar{x}_{i}-\bar{x}_{1}\right|^{2}} \leq \frac{a_{1}}{j^{2}}
$$

for some constant $a_{1} \geq a_{0}>0$, which implies

$$
\frac{a_{0}^{\prime}}{j^{N}}+O\left(\frac{d}{j^{N-2}}\right) \leq \frac{1}{k^{N-2}} G\left(\bar{x}_{j}, \bar{x}_{1}\right) \leq \frac{a_{1}^{\prime}}{j^{N}}+O\left(\frac{d}{j^{N-2}}\right)
$$

for some constant $a_{1}^{\prime} \geq a_{0}^{\prime}>0$. Hence, there is a constant $B_{4}>0$, such that

$$
\sum_{j=2}^{k} G\left(\bar{x}_{j}, \bar{x}_{1}\right)=k^{N-2}\left(\frac{B_{4}}{\left|\bar{x}_{1}\right|^{N-2}}+O\left(\frac{1}{k^{N-1}}\right)+O(d)\right)=B_{4} k^{N-2}+O\left(k^{N-2} d\right) .
$$

Thus, we obtain that there are positive constants $A_{1}, A_{2}$ and $A_{3}$, such that

$$
\begin{aligned}
F(d, \Lambda) & =k\left(A+\frac{A_{1}}{\Lambda^{N-2} \mu^{N-2} d^{N-2}}+A_{2} d-\frac{A_{3} k^{N-2}}{\Lambda^{N-2} \mu^{N-2}}+O\left(\frac{1}{\mu^{1+\sigma}}\right)\right), \\
\frac{\partial F(d, \Lambda)}{\partial \Lambda} & =k\left(-\frac{A_{1}(N-2)}{\Lambda^{N-1} \mu^{N-2} d^{N-2}}+\frac{A_{3}(N-2) k^{N-2}}{\Lambda^{N-1} \mu^{N-2}}+O\left(\frac{1}{\mu^{1+\sigma}}\right)\right), \\
\frac{\partial F(d, \Lambda)}{\partial d} & =k\left(-\frac{A_{1}(N-2)}{\Lambda^{N-2} \mu^{N-2} d^{N-1}}+A_{2}+O\left(\frac{1}{\mu^{\sigma}}\right)\right),
\end{aligned}
$$

Note that $d=1-r / \mu$, and $\mu=k^{\frac{N-1}{N-2}}$. Define $D=d / k$. Then, from (3.2) and (3.3), $\partial F(d, \Lambda) / \partial \Lambda=0$ and $\partial F(d, \Lambda) / \partial d=0$ are equivalent to

$$
-\frac{A_{1}(N-2)}{\Lambda^{N-1} D^{N-2}}+\frac{A_{3}(N-2)}{\Lambda^{N-1}}+O\left(\frac{1}{\mu^{\sigma}}\right)=0,
$$


and

$$
-\frac{A_{1}(N-2)}{\Lambda^{N-2} D^{N-1}}+A_{2}+O\left(\frac{1}{\mu^{\sigma}}\right)=0
$$

respectively.

Proof of Theorem 1.2. Let

$$
f_{1}(D, \Lambda)=-\frac{A_{1}(N-2)}{\Lambda^{N-1} D^{N-2}}+\frac{A_{3}(N-2)}{\Lambda^{N-1}} \quad \text { and } \quad f_{2}(D, \Lambda)=-\frac{A_{1}(N-2)}{\Lambda^{N-2} D^{N-1}}+A_{2} .
$$

Then, $f_{1}=0$ and $f_{2}=0$ have a unique solution

$$
D_{0}=\left(\frac{A_{1}}{A_{3}}\right)^{1 /(N-2)}, \quad \Lambda_{0}=\left(\frac{A_{1}(N-2)}{A_{2} D_{0}^{N-1}}\right)^{1 /(N-2)} .
$$

On the other hand, it is easy to see that

$$
\frac{\partial f_{1}\left(D_{0}, \Lambda_{0}\right)}{\partial \Lambda}=0, \quad \frac{\partial f_{2}\left(D_{0}, \Lambda_{0}\right)}{\partial D}>0, \quad \text { and } \quad \frac{\partial f_{1}\left(D_{0}, \Lambda_{0}\right)}{\partial D}=\frac{\partial f_{2}\left(D_{0}, \Lambda_{0}\right)}{\partial \Lambda}>0 .
$$

Thus the linear operator of $f_{1}=0$ and $f_{2}=0$ at $\left(D_{0}, \Lambda_{0}\right)$ is invertible. As a result, (3.4) and (3.5) have a solution near $\left(D_{0}, \Lambda_{0}\right)$.

\section{A. Energy expansion}

In both appendices, we always assume that

$$
x_{j}=\left(r \cos \frac{2(j-1) \pi}{k}, r \sin \frac{2(j-1) \pi}{k}, 0\right), \quad j=1, \ldots, k,
$$

where 0 is the zero vector in $\mathbb{R}^{N-2}$, and $r \in\left[\mu\left(1-r_{0} / k\right), \mu\left(1-r_{1} / k\right)\right]$. Let

$$
\bar{x}_{j}=\frac{1}{\mu} x_{j} .
$$

Let $G(y, z)$ be the Green function of $-\Delta$ in $B_{1}(0)$ with the Dirichlet boundary condition. Let $H(y, z)$ be the regular part of the Green function.

Recall that

$$
\begin{gathered}
\mu=k^{\frac{N-1}{N-2}} \\
I(u)=\frac{1}{2} \int_{B_{\mu}(0)}|D u|^{2}-\frac{1}{2^{*}} \int_{B_{\mu}(0)} K\left(\frac{|y|}{\mu}\right)|u|^{2^{*}}, \\
U_{x_{j}, \Lambda}(y)=(N(N-2))^{(N-2) / 4} \frac{\Lambda^{(N-2) / 2}}{\left(1+\Lambda^{2}\left|y-x_{j}\right|^{2}\right)^{(N-2) / 2}},
\end{gathered}
$$

and

$$
W_{r, \Lambda}(y)=\sum_{j=1}^{k} P U_{x_{j}, \Lambda}(y)
$$


where $P U_{x, \Lambda}$ is the solution of (1.5). It is well known that

$$
U_{x_{j}, \Lambda}(y)-P U_{x_{j}, \Lambda}(y)=\frac{H(\bar{y}, \bar{x})}{\mu^{N-2}}+O\left(\frac{1}{d^{N} \mu^{N}}\right),
$$

where $d=1-|\bar{x}|=1-|x| / \mu$.

In this section, we will calculate $I\left(W_{r, \Lambda}\right)$.

Proposition A.1. We have

$$
I\left(W_{r, \Lambda}\right)=k\left(A+\frac{B_{1} H\left(\bar{x}_{1}, \bar{x}_{1}\right)}{\Lambda^{N-2} \mu^{N-2}}+B_{2} K^{\prime}(1) d-\sum_{i=2}^{k} \frac{B_{1} G\left(\bar{x}_{i}, \bar{x}_{1}\right)}{\Lambda^{N-2} \mu^{N-2}}+O\left(\frac{1}{\mu^{1+\sigma}}\right)\right),
$$

where $A, B_{1}$ and $B_{2}$ are positive constants.

Proof. By using the assumed symmetry, we have

$$
\begin{aligned}
& \int_{B_{\mu}(0)}\left|D W_{r, \Lambda}\right|^{2}=\sum_{j=1}^{k} \sum_{i=1}^{k} \int_{B_{\mu}(0)} U_{x_{j}, \Lambda}^{2^{*}-1} P U_{x_{i}, \Lambda} \\
& =k\left(\int_{B_{\mu}(0)} U_{0,1}^{2^{*}}-\int_{B_{\mu}(0)} U_{x_{1}, \Lambda}^{2^{*}-1}\left(U_{x_{1}, \Lambda}-P U_{x_{1}, \Lambda}\right)+\sum_{i=2}^{k} \int_{B_{\mu}(0)} U_{x_{1}, \Lambda}^{2^{*}-1} P U_{x_{i}, \Lambda}\right) \\
& =k\left(\int_{\mathbb{R}^{N}} U^{2^{*}}-\frac{\bar{B}_{1} H\left(\bar{x}_{1}, \bar{x}_{1}\right)}{\Lambda^{N-2} \mu^{N-2}}+\frac{\bar{B}_{1} \sum_{i=2}^{k} G\left(\bar{x}_{i}, \bar{x}_{1}\right)}{\Lambda^{N-2} \mu^{N-2}}+O\left(\frac{1}{\mu^{N /(N-1)}}\right)\right),
\end{aligned}
$$

where

$$
\bar{B}_{1}=\int_{\mathbb{R}^{N}} U^{2^{*}-1}
$$

Let

$$
\Omega_{j}=\left\{y: y=\left(y^{\prime}, y^{\prime \prime}\right) \in B_{\mu}(0),\left\langle\frac{y^{\prime}}{\left|y^{\prime}\right|}, \frac{x_{j}}{\left|x_{j}\right|}\right\rangle \geq \cos \frac{\pi}{k}\right\}
$$

Then,

$$
\begin{aligned}
\int_{B_{\mu}(0)} K\left(\frac{|y|}{\mu}\right)\left|W_{r, \Lambda}\right|^{2^{*}}=k \int_{\Omega_{1}} K\left(\frac{|y|}{\mu}\right)\left|W_{r, \Lambda}\right|^{2^{*}} \\
=k\left(\int_{\Omega_{1}} K\left(\frac{|y|}{\mu}\right)\left(P U_{x_{1}, \Lambda}\right)^{2^{*}}-2^{*} \int_{\Omega_{1}} \sum_{i=2}^{k}\left(P U_{x_{1}, \Lambda}\right)^{2^{*}-1} P U_{x_{i}, \Lambda}\right. \\
\left.\quad+O\left(\int_{\Omega_{1}}\left|K\left(\frac{|y|}{\mu}\right)-1\right| \sum_{i=2}^{k} U_{x_{1}, \Lambda}^{2^{*}-1} U_{x_{i}, \Lambda}+\int_{\Omega_{1}} U_{x_{1}, \Lambda}^{2^{*} / 2}\left(\sum_{i=2}^{k} U_{x_{i}, \Lambda}\right)^{2^{*} / 2}\right)\right) .
\end{aligned}
$$

Note that for $y \in \Omega_{1},\left|y-x_{i}\right| \geq\left|y-x_{1}\right|$. Using (2.18), we find that for any $t \in(1, N-2)$,

$$
\sum_{i=2}^{k} U_{x_{i}, \Lambda} \leq \frac{C}{\left(1+\left|y-x_{1}\right|\right)^{N-2-t}} \sum_{i=2}^{k} \frac{1}{\left|x_{i}-x_{1}\right|^{t}}
$$


If we take the constant $t$ close to $N-2$, then

$$
\int_{\Omega_{1}} U_{x_{1}, \Lambda}^{2^{*} / 2}\left(\sum_{i=2}^{k} U_{x_{i}, \Lambda}\right)^{2^{*} / 2}=O\left(\left(\frac{k}{\mu}\right)^{t \frac{N}{N-2}}\right)=O\left(\frac{1}{\mu^{1+\sigma}}\right) .
$$

On the other hand, it is easy to show that

$$
\begin{aligned}
\int_{\Omega_{1}} \sum_{i=2}^{k}\left(P U_{x_{1}, \Lambda}\right)^{2^{*}-1} P U_{x_{i}, \Lambda} & =\frac{\bar{B}_{2} G\left(\bar{x}_{i}, \bar{x}_{1}\right)}{\Lambda^{N-2} \mu^{N-2}}+O\left(\frac{k^{N}}{\mu^{N}}\right) \\
& =\frac{\bar{B}_{2} G\left(\bar{x}_{i}, \bar{x}_{1}\right)}{\Lambda^{N-2} \mu^{N-2}}+O\left(\frac{1}{\mu^{1+\sigma}}\right)
\end{aligned}
$$

and

$$
\int_{\Omega_{1}}\left|K\left(\frac{|y|}{\mu}\right)-1\right| \sum_{i=2}^{k} U_{x_{1}, \Lambda}^{2^{*}-1} U_{x_{i}, \Lambda}=O\left(\frac{1}{\mu^{1+\sigma}}\right) .
$$

Moreover,

$$
\begin{aligned}
& \int_{\Omega_{1}} K\left(\frac{|y|}{\mu}\right)\left(P U_{x_{1}, \Lambda}\right)^{2^{*}} \\
& =\int_{\Omega_{1}}\left(P U_{x_{1}, \Lambda}\right)^{2^{*}}+\int_{\Omega_{1}}\left(K\left(\frac{|y|}{\mu}\right)-1\right) U_{x_{1}, \Lambda}^{2^{*}}+O\left(\int_{\Omega_{1}}\left|K\left(\frac{|y|}{\mu}\right)-1\right| U_{x_{1}, \Lambda}^{2^{*}-1} \frac{H\left(y, x_{1}\right)}{\mu^{N-2}}\right) \\
& =\int_{\mathbb{R}^{N}} U^{2^{*}}-2^{*} \frac{\bar{B}_{1} H\left(\bar{x}_{1}, \bar{x}_{1}\right)}{\Lambda^{N-2} \mu^{N-2}}+\int_{\Omega_{1}}\left(K\left(\frac{|y|}{\mu}\right)-1\right) U_{x_{1}, \Lambda}^{2^{*}}+O\left(\frac{1}{\mu^{1+\sigma}}\right) .
\end{aligned}
$$

However,

$$
\begin{aligned}
\int_{\Omega_{1}}\left(K\left(\frac{|y|}{\mu}\right)-1\right) & U_{x_{1}, \Lambda}^{2^{*}}=\left(K\left(\left|\bar{x}_{1}\right|\right)-1\right) \int_{\mathbb{R}^{N}} U^{2^{*}}+O\left(\frac{1}{\mu^{2}}\right) \\
& =-K^{\prime}(1) d \int_{\mathbb{R}^{N}} U^{2^{*}}+O\left(d^{2}\right)=-K^{\prime}(1) d \int_{\mathbb{R}^{N}} U^{2^{*}}+O\left(\frac{1}{\mu^{1+\sigma}}\right) .
\end{aligned}
$$

Thus, we have proved

$$
\begin{aligned}
\int_{\mathbb{R}^{N}} K\left(\frac{|y|}{\mu}\right)\left|W_{r, \Lambda}\right|^{2^{*}}= & k\left(\int_{\mathbb{R}^{N}} U^{2^{*}}-K^{\prime}(1) d \int_{\mathbb{R}^{N}} U^{2^{*}}-2^{*} \frac{\bar{B}_{1} H\left(\bar{x}_{1}, \bar{x}_{1}\right)}{\Lambda^{N-2} \mu^{N-2}}\right. \\
& \left.+2^{*} \sum_{i=2}^{k} \frac{\bar{B}_{1} G\left(\bar{x}_{i}, \bar{x}_{1}\right)}{\Lambda^{N-2} \mu^{N-2}}+O\left(\frac{1}{\mu^{1+\sigma}}\right)\right) .
\end{aligned}
$$

We also need to calculate

$$
\frac{\partial I\left(W_{r, \Lambda}\right)}{\partial \Lambda} \text { and } \frac{\partial I\left(W_{r, \Lambda}\right)}{\partial r} .
$$


Proposition A.2. We have

$$
\frac{\partial I\left(W_{r, \Lambda}\right)}{\partial \Lambda}=k(N-2) B_{1}\left(-\frac{H\left(\bar{x}_{1}, \bar{x}_{1}\right)}{\Lambda^{N-1} \mu^{N-2}}+\sum_{i=2}^{k} \frac{G\left(\bar{x}_{1}, \bar{x}_{i}\right)}{\Lambda^{N-1} \mu^{N-2}}+O\left(\frac{1}{\mu^{1+\sigma}}\right)\right),
$$

and

$$
\frac{\partial I\left(W_{r, \Lambda}\right)}{\partial r}=k\left(B_{1} \frac{\frac{\partial H\left(\bar{x}_{1}, \bar{x}_{1}\right)}{\partial r}}{\Lambda^{N-2} \mu^{N-2}}-B_{2} K^{\prime}(1) \frac{1}{\mu}-\sum_{i=2}^{k} \frac{B_{1} \frac{\partial G\left(\bar{x}_{1}, \bar{x}_{i}\right)}{\partial r}}{\Lambda^{N-1} \mu^{N-2}}+O\left(\frac{1}{\mu^{1+\sigma}}\right)\right),
$$

where $B_{1}$ is the same positive constant as in Proposition A.1.

Proof. We use $\partial$ to denote either $\partial / \partial \Lambda$ or $\partial / \partial r$. Using the symmetry, we have

$$
\begin{gathered}
\partial I\left(W_{r, \Lambda}\right)=k\left(\left(2^{*}-1\right) \sum_{i=2}^{k} \int_{\mathbb{R}^{N}} U_{x_{1}, \Lambda}^{2^{*}-2} \partial\left(U_{x_{1}, \Lambda}\right) P U_{x_{i}, \Lambda}\right. \\
\left.-\int_{\Omega_{1}} K\left(\frac{|y|}{\mu}\right) W_{r, \Lambda}^{2^{*}-1} \partial W_{r, \Lambda}\right) .
\end{gathered}
$$

Then the proof of this proposition is similar to the proof of Proposition A.1, so we omit it.

\section{B. Basic estimates}

In this section, we list some lemmas, whose proofs can be found in [15].

For each fixed $i$ and $j, i \neq j$, consider the function

$$
g_{i j}(y)=\frac{1}{\left(1+\left|y-x_{j}\right|\right)^{\alpha}} \frac{1}{\left(1+\left|y-x_{i}\right|\right)^{\beta}},
$$

where $\alpha \geq 1$ and $\beta \geq 1$ are constants.

Lemma B.1. For any constant $0<\sigma \leq \min (\alpha, \beta)$, there is a constant $C>0$ such that

$$
g_{i j}(y) \leq \frac{C}{\left|x_{i}-x_{j}\right|^{\sigma}}\left(\frac{1}{\left(1+\left|y-x_{i}\right|\right)^{\alpha+\beta-\sigma}}+\frac{1}{\left(1+\left|y-x_{j}\right|\right)^{\alpha+\beta-\sigma}}\right) .
$$

Lemma B.2. For any constant $0<\sigma<N-2$, there is a constant $C>0$ such that

$$
\int_{\mathbb{R}^{N}} \frac{1}{|y-z|^{N-2}} \frac{1}{(1+|z|)^{2+\sigma}} d z \leq \frac{C}{(1+|y|)^{\sigma}} .
$$

Recall that

$$
W_{r, \Lambda}(y)=\sum_{j=1}^{k} P U_{x_{j}, \Lambda}
$$


Lemma B.3. Suppose that $N \geq 4$. Then there is a small $\theta>0$, such that

$$
\begin{aligned}
\int_{\mathbb{R}^{N}} \frac{1}{|y-z|^{N-2}} W_{r, \Lambda}^{4 /(N-2)}(z) & \sum_{j=1}^{k} \frac{1}{\left(1+\left|z-x_{j}\right|\right)^{\frac{N-2}{2}+\tau}} d z \\
& \leq C \sum_{j=1}^{k} \frac{1}{\left(1+\left|y-x_{j}\right|\right)^{\frac{N-2}{2}+\tau+\theta}}
\end{aligned}
$$

Proof. The proof can be found in [15]. We only need to use

$$
W_{r, \Lambda}(y) \leq C \sum_{i=1}^{k} \frac{1}{\left(1+\left|y-x_{i}\right|\right)^{N-2}}
$$

\section{References}

[1] Byeon, J. And Wang, Z.-Q.: On the Hénon equation: asymptotic profile of ground states. I. Ann. Inst. H. Poincaré Anal. Non Linéaire 23 (2006), 803-828.

[2] Byeon, J. And WAng, Z.-Q.: On the Hénon equation: asymptotic profile of ground states. II. J. Differential Equations 216 (2005), 78-108.

[3] CaO, D. And Peng, S.: The asymptotic behaviour of the ground state solutions for Hénon equation. J. Math. Anal. Appl. 278 (2003), 1-17.

[4] CaO, D., Peng, S. And Yan, S.: Asymptotic behaviour of ground state solutions for the Hénon equation. IMA J. Appl. Math. 74 (2009), 468-480.

[5] del Pino, M., Felmer, P. And Musso, M.: Two-bubble solutions in the supercritical Bahri-Coron's problem. Calc. Var. Partial Differential Equations 16 (2003), 113-145.

[6] Druet, O. and Laurain, P.: Private communication.

[7] HÉnon, M.: Numerical experiments on the stability of spherical stellar systems. Astronom. and Astrophys. 24 (1973), 229-238.

[8] Hirano, N.: Existence of positive solutions for the Hénon equation involving critical Sobolev terms. J. Differential Equations 247 (2009), 1311-1333.

[9] Ni, W.-M.: A nonlinear Dirichlet problem on the unit ball and its applications. Indiana Univ. Math. J. 31 (1982), 801-807.

[10] Peng, S.: Multiple boundary concentrating solutions to Dirichlet problem of Hénon equation. Acta Math. Appl. Sin. Engl. Ser. 22 (2006), 137-162.

[11] Pistoia, A. And Serra, E.: Multi-peak solutions for the Hénon equation with slightly subcritical growth. Math. Z. 256 (2007), 75-97.

[12] Serra, E.: Non-radial positive solutions for the Hénon equation with critical growth. Cal. Var. Partial Differential Equations 23 (2005), no. 3, 301-326.

[13] Smets, D., Su, J. And Willem, M.: Non-radial ground states for the Hénon equation. Commun. Contemp. Math. 4 (2002), no. 3, 467-480. 
[14] Smets, D. And Willem, M.: Partial symmetry and asymptotic behavior for some elliptic variational problems. Calc. Var. Partial Differential Equations 18 (2003), $57-75$.

[15] WeI, J. AND YAN, S.: Infinitely many solutions for the prescribed scalar curvature problem on $\mathbb{S}^{N}$. J. Funct. Anal. 258 (2010), no. 9, 3048-3081.

Received December 1, 2011.

Juncheng Wei: Department of Mathematics, The Chinese University of Hong Kong, Shatin, Hong Kong.

E-mail: wei@math.cuhk.edu.hk

Shusen Yan: Department of Mathematics, The University of New England, Armidale, NSW 2351, Australia.

E-mail: syan@turing.une.edu.au

The first author is supported by an Earmarked Grant from the RGC of Hong Kong, a direct grant from the CUHK and an Oversea Joint Research Grant of the NSFC. The second author is partially supported by the ARC. The first author thanks the Institute EHESS for its hospitality during his stay in June, 2009. 